Please cite this paper as follows:

Han-Chin Wu and Hong-Ki Hong, Endochronic Description of Plastic Anisotropy in Sheet Metal, International Journal of Solids and Structures, Vol.36, pp.2735-2756, 1999. 


\title{
Endochronic description of plastic anisotropy in sheet metal Han-Chin $\mathrm{Wu}^{\mathrm{a}, *}$, Hong-Ki Hong ${ }^{\mathrm{b}}$ \\ a Department of Civil and Environmental Engineering, The University of Iowa, Iowa City, Iowa, U.S.A. ${ }^{\mathrm{b}}$ Department of Civil Engineering, National Taiwan University, Taipei, Taiwan, R.O.C.
}

Received 24 June 1997; in revised form 1 April 1998

\begin{abstract}
It is shown in this paper that an extended form of Hill's quadratic yield criterion for anisotropic sheet metal can be derived from an endochronic theory of plasticity. The extended form considers the combined isotropic-kinematic hardening and the 'anomalous behavior' observed in the anisotropic plastic behavior of sheet metals can be accounted for by the concept of kinematic hardening.

This form of anisotropic endochronic theory can accommodate the usual requirement of normality between the plastic strain rate and the yield function. In addition, the theory leads naturally to the expressions for back stresses. This work provides an additional example to show that the form of the intrinsic time is directly related to the form of the yield function.

It is suggested that the coefficients of the quadratic yield function be determined from the yield stresses obtained from a set of tension tests. (C) 1999 Elsevier Science Ltd. All rights reserved.
\end{abstract}

\section{Introduction}

In the case of rolled sheet metal, when principal axes of anisotropy are the axes of reference, the yield function $\phi$ proposed by Hill (1948) has been widely used. The yield function $\phi$ is

$$
2 \phi=(G+H) \sigma_{x}^{2}-2 H \sigma_{x} \sigma_{y}+(F+H) \sigma_{y}^{2}+2 N \sigma_{x y}^{2}=1
$$

where $\left(\sigma_{x}, \sigma_{y}, \sigma_{x y}\right)$ are the in-plane components of Cauchy stress; and the out-of-plane components are considered to be zero. The coefficients $F, G, H$ and $N$ specify the anisotropy of the metal sheet. This quadratic form (and its variations such as the one for planar isotropy) has been repeatedly used in applications. It is generally satisfactory for predicting the sheet metal behavior for $R \geqslant 1$, where $R$ is the plastic strain ratio of the transverse to the thickness strain and it specifies anisotropy. In the case of $R<1$, 'anomalous behavior' has been observed by Pearce (1968) and Woodthorpe and Pearce (1970) for commercially pure aluminum sheet. In this case, the yield stress in the

\footnotetext{
* Corresponding author. Fax: $001319 / 3355660$
} 
equibiaxial tension test is higher than that for the uniaxial tension, and eqn (1) is known not to predict this behavior.

It was pointed out by Wu et al. (1998) that the 'anomalous behavior' was the consequence of neglecting the role played by kinematic hardening in most anisotropic theory of sheet metals. Another cause leading to this doubtful result is by expressing the coefficients of yield function, i.e. $F, G, H$ and $N$ in eqn (1), in terms of the strain-ratio $R$. The latter is equivalent to expressing the yield function in terms of the flow rule. This, of course, is not in accord with the conventional theory of plasticity. Wu et al. (1998) showed that by considering a combined isotropic-kinematic hardening behavior, an extended form of eqn (1), i.e.,

$$
(G+H)\left(\sigma_{x}-r_{x}\right)^{2}-2 H\left(\sigma_{x}-r_{x}\right)\left(\sigma_{y}-r_{y}\right)+(H+F)\left(\sigma_{y}-r_{y}\right)^{2}+2 N\left(\sigma_{x y}-r_{x y}\right)^{2}=f^{2}
$$

is useful for predicting the sheet metal behavior for all values of $R$-ratio. In (2), $r_{x}, r_{y}$ and $r_{x y}$ specify the center of the yield surface and therefore, represent the kinematic hardening; $f$ represents the isotropic hardening. Thus, the more complex non-quadratic forms of yield function, such as Hill (1979, 1990), Gotoh (1977), Barlat and Richmond (1987) and Barlat and Lian (1989), are not needed.

In addition to yield function, eqn (2), flow rule and hardening rules are required for plasticity. These are separately proposed rules, although some of the parameters may be inter-related. In this paper, the endochronic theory is used to derive all equations, i.e., yield function, flow rule and hardening rules, by use of a unified approach which is based on irreversible thermodynamics of internal state variables.

The endochronic theory of plasticity was initially proposed by Valanis $(1971,1980)$. Further development of the theory in the case of initially isotropic materials was due to $\mathrm{Wu}$ and Yip (1980, 1981), Wu and Yang (1983), Valanis and Lee (1984), Im and Atluri (1987), Wu et al. (1995b) and others. The case of deformation induced anisotropy was investigated by $\mathrm{Wu}$ and Yeh (1987), Wu and $\mathrm{Lu}$ (1995) and $\mathrm{Wu}$ et al. (1995a). In these papers, the distortion of the yield surface was considered together with the combined isotropic-kinematic hardening. It was shown in $\mathrm{Wu}$ et al. (1995a) that the form of the yield function depends closely upon the expressions used to define the intrinsic time, which is a time-like parameter used to register the history of deformation in the endochronic theory. This idea is further explored in the present paper. Also, in the works of $\mathrm{Wu}$ and Yeh (1987), Wu and $\mathrm{Lu}$ (1995) and $\mathrm{Wu}$ et al. (1995a), the plastic strain rate is necessarily pointing along the radial direction, emanating from the center of the yield surface, and it is not normal to the yield surface after the yield surface has suffered a distortion. In this paper, it is shown that it is possible to formulate an anisotropic endochronic theory that obeys the normality rule, if the yield surface is expressed in a quadratic form given by (2). The present paper addresses the problem of initial anisotropy together with the deformation induced anisotropy.

\section{The endochronic constitutive framework}

In the formulation by use of Helmholtz free energy $\psi$, see Valanis (1975), the free energy is a function of the current strain $\varepsilon_{i j}$ and $n$ number of internal state variables $q_{i j}^{r}$, where $r=1,2, \ldots, n$. The internal state variables are phenomenological variables used to specify the current state of material internal structure and the free energy is 


$$
\psi=\frac{1}{2} \sum_{r} A_{i j k m}^{r}\left(\varepsilon_{i j}-q_{i j}^{r}\right)\left(\varepsilon_{k m}-q_{k m}^{r}\right)
$$

where $A_{i j k m}^{r}$ are constants.

For rolled metal sheet, let $x$ denote the rolling direction (RD); $y$ the transverse direction (TD); and $z$ the normal direction (ND). The nonzero strain components are $\varepsilon_{x}, \varepsilon_{y}, \varepsilon_{x y}$ and $\varepsilon_{z}$. No energy is stored due to $\varepsilon_{z}$. Thus, (3) reduces to

$$
\psi=\frac{1}{2} \sum_{r}\left[A^{r}\left(\varepsilon_{x}-q_{x}^{r}\right)^{2}+B^{r}\left(\varepsilon_{y}-q_{y}^{r}\right)^{2}+2 D^{r}\left(\varepsilon_{x y}-q_{x y}^{r}\right)^{2}+2 C^{r}\left(\varepsilon_{x}-q_{x}^{r}\right)\left(\varepsilon_{y}-q_{y}^{r}\right)\right]
$$

where $A^{r}, B^{r}, C^{r}$ and $D^{r}$ are constants. For a stable material, any deformation will cause the free energy density $\psi$ to increase. A consideration of uniaxial straining in the $x$-direction leads to $A^{r}>0$ and a consideration of uniaxial straining in the $y$-direction leads to $B^{r}>0$. Finally, pure shear leads to $D^{r}>0$. The stress components are

$$
\begin{aligned}
& \sigma_{x}=\frac{\partial \psi}{\partial \varepsilon_{x}}=\sum_{r}\left[A^{r}\left(\varepsilon_{x}-q_{x}^{r}\right)+C^{r}\left(\varepsilon_{y}-q_{y}^{r}\right)\right] \\
& \sigma_{y}=\frac{\partial \psi}{\partial \varepsilon_{y}}=\sum_{r}\left[B^{r}\left(\varepsilon_{y}-q_{y}^{r}\right)+C^{r}\left(\varepsilon_{x}-q_{x}^{r}\right)\right] \\
& \sigma_{x y}=\frac{\partial \psi}{\partial \varepsilon_{x y}}=\sum_{r}\left[D^{r}\left(\varepsilon_{x y}-q_{x y}^{r}\right)\right]
\end{aligned}
$$

and the evolution equations for the internal variables are

$$
\frac{\partial \psi}{\partial q_{i j}^{r}}+b_{i j k m}^{r} \frac{\mathrm{d} q_{k m}^{r}}{\mathrm{~d} z}=0 \quad(r \text { not summed })
$$

where $b_{i j k m}^{r}$ is the dissipation tensor representing the viscosity of the material. The evolution of the variables is with respect to a time-like parameter $z$ which is often referred to as the intrinsic time or the endochronic time. The intrinsic time is monotonically increasing and is defined in terms of the plastic strain. The intrinsic time measure for sheet metals will be further discussed in a later section. In the rolled sheet metal, the components of internal variables are $\left(q_{x}^{r}, q_{y}^{r}, q_{x y}^{r}\right)$ and it is assumed that there are no coupling effects among these components, so that the dissipation tensor has the following form

$$
\underset{\approx}{b^{r}}=\left[\begin{array}{ccc}
b_{x}^{r} & 0 & 0 \\
0 & b_{y}^{r} & 0 \\
0 & 0 & b_{x y}^{r}
\end{array}\right]
$$

Using (4) and (7), (6) reduces to

$$
\frac{\mathrm{d} q_{x}^{r}}{\mathrm{~d} z}+P^{r} q_{x}^{r}+U^{r} q_{y}^{r}=P^{r} \varepsilon_{x}+U^{r} \varepsilon_{y}
$$




$$
\begin{aligned}
& \frac{\mathrm{d} q_{y}^{r}}{\mathrm{~d} z}+Q^{r} q_{y}^{r}+V^{r} q_{x}^{r}=Q^{r} \varepsilon_{y}+V^{r} \varepsilon_{x} \\
& \frac{\mathrm{d} q_{x y}^{r}}{\mathrm{~d} z}+R^{r} q_{x y}^{r}=R^{r} \varepsilon_{x y}
\end{aligned}
$$

where

$$
P^{r}=A^{r} / b_{x}^{r}, U^{r}=C^{r} / b_{x}^{r}, Q^{r}=B^{r} / b_{y}^{r}, V^{r}=C^{r} / b_{y}^{r} \quad \text { and } \quad R^{r}=D^{r} / b_{x y}^{r}
$$

Note that (8a) and (b) are coupled in $q_{x}^{r}$ and $q_{y}^{r}$. A standard procedure may be used to decouple the equations. The resulting equations are

$$
\begin{aligned}
& \frac{\mathrm{d} \bar{q}_{x}^{r}}{\mathrm{~d} z}+\lambda_{1}^{r} \bar{q}_{x}^{r}={\widetilde{C^{r}}}^{r} \varepsilon_{x}+\widetilde{D}^{r} \varepsilon_{y} \\
& \frac{\mathrm{d} \bar{q}_{y}^{r}}{\mathrm{~d} z}+\lambda_{2}^{r} \bar{q}_{y}^{r}=\widetilde{E}^{r} \varepsilon_{x}+\tilde{F}^{r} \varepsilon_{y}
\end{aligned}
$$

where $\lambda_{1}^{r}$ and $\lambda_{2}^{r}$ are eigenvalues of the matrix $\left[\begin{array}{ll}P^{r} & U^{r} \\ V^{r} & Q^{r}\end{array}\right]$. Note that since $U^{r} \neq 0$ and $V^{r} \neq 0$, there are always two real eigenvalues. $\bar{q}_{x}^{r}$ and $\bar{q}_{y}^{r}$ are related to $q_{x}^{r}$ and $q_{y}^{r}$ through the eigenvectors of the matrix by the following relations

$$
\begin{aligned}
& q_{x}^{r}=U^{r}\left(\bar{q}_{x}^{r}+\bar{q}_{y}^{r}\right) \\
& q_{y}^{r}=\left(-P^{r}+\lambda_{1}^{r}\right) \bar{q}_{x}^{r}+\left(-P^{r}+\lambda_{2}^{r}\right) \bar{q}_{y}^{r}
\end{aligned}
$$

and

$$
\begin{aligned}
\widetilde{C}^{r} & =\left(-\left(P^{r}\right)^{2}+\lambda_{2}^{r} P^{r}-P^{r} V^{r}+\lambda_{1}^{r} V^{r}\right) / U^{r}\left(\lambda_{2}^{r}-\lambda_{1}^{r}\right) \\
\widetilde{D}^{r} & =\left(-P^{r} U^{r}+\lambda_{2}^{r} U^{r}-P^{r} Q^{r}+\lambda_{1}^{r} Q^{r}\right) / U^{r}\left(\lambda_{2}^{r}-\lambda_{1}^{r}\right) \\
\widetilde{E}^{r} & =\left(U^{r} P^{r}+U^{r} V^{r}\right) / U^{r}\left(\lambda_{2}^{r}-\lambda_{1}^{r}\right) \\
\widetilde{F}^{r} & =\left(\left(U^{r}\right)^{2}+U^{r} Q^{r}\right) / U^{r}\left(\lambda_{2}^{r}-\lambda_{1}^{r}\right)
\end{aligned}
$$

Note that (10) are now decoupled in $\bar{q}_{x}^{r}$ and $\bar{q}_{y}^{r}$. These equations may be integrated with results substituted into (11) to obtain

$$
q_{x}^{r}=U^{r} \int_{0}^{z} \mathrm{e}^{-\lambda_{1}^{r}\left(z-z^{\prime}\right)}\left[\tilde{C}^{r} \varepsilon_{x}\left(z^{\prime}\right)+\tilde{D}^{r} \varepsilon_{y}\left(z^{\prime}\right)\right] \mathrm{d} z^{\prime}+U^{r} \int_{0}^{z} \mathrm{e}^{-\lambda_{2}^{r}\left(z-z^{\prime}\right)}\left[\tilde{E}^{r} \varepsilon_{x}\left(z^{\prime}\right)+\tilde{F}^{r} \varepsilon_{y}\left(z^{\prime}\right)\right] \mathrm{d} z^{\prime}
$$




$$
\begin{aligned}
q_{y}^{r}=\left(-P^{r}+\lambda_{1}^{r}\right) \int_{0}^{z} \mathrm{e}^{-\lambda_{1}^{r}\left(z-z^{\prime}\right)}\left[\tilde{C}^{r} \varepsilon_{x}\left(z^{\prime}\right)\right. & \left.+\tilde{D}^{r} \varepsilon_{y}\left(z^{\prime}\right)\right] \mathrm{d} z^{\prime} \\
& +\left(-P^{r}+\lambda_{2}^{r}\right) \int_{0}^{z} \mathrm{e}^{-\lambda_{2}^{r}\left(z-z^{\prime}\right)}\left[\widetilde{E}^{r} \varepsilon_{x}\left(z^{\prime}\right)+\tilde{F}^{r} \varepsilon_{y}\left(z^{\prime}\right)\right] \mathrm{d} z^{\prime}
\end{aligned}
$$

Also, (8c) may be integrated to yield

$$
q_{x y}^{r}=R^{r} \int_{0}^{z} \mathrm{e}^{-R^{r}\left(z-z^{\prime}\right)} \varepsilon_{x y}\left(z^{\prime}\right) \mathrm{d} z^{\prime}
$$

with $q_{x}^{r}(0)=q_{y}^{r}(0)=q_{x y}^{r}(0)=0$.

Substitution of (13) into (5) and by use of integration by parts, the following expressions are found

$$
\begin{aligned}
& \begin{aligned}
& \sigma_{x}=Y_{1} \varepsilon_{x}(z)+Y_{2} \varepsilon_{y}(z)+\sum_{r}\left\{M^{r} \int_{0}^{z} \mathrm{e}^{-\lambda_{1}^{\prime}\left(z-z^{\prime}\right)}\right. {\left.\left[\widetilde{C^{r}} \frac{\mathrm{d} \varepsilon_{x}}{\mathrm{~d} z^{\prime}}+\widetilde{D^{r}} \frac{\mathrm{d} \varepsilon_{y}}{\mathrm{~d} z^{\prime}}\right] \mathrm{d} z^{\prime}\right\} } \\
&+\sum_{r}\left\{N^{r} \int_{0}^{z} \mathrm{e}^{-\lambda_{2}^{r}\left(z-z^{\prime}\right)}\left[\widetilde{E}^{r} \frac{\mathrm{d} \varepsilon_{x}}{\mathrm{~d} z^{\prime}}+\widetilde{F}^{r} \frac{\mathrm{d} \varepsilon_{y}}{\mathrm{~d} z^{\prime}}\right] \mathrm{d} z^{\prime}\right\} \\
& \sigma_{y}=Y_{3} \varepsilon_{y}(z)+Y_{4} \varepsilon_{x}(z)+\sum_{r}\left\{K^{r} \int_{0}^{z} \mathrm{e}^{-\lambda_{1}^{r}\left(z-z^{\prime}\right)}\right. {\left.\left[\widetilde{C^{r}} \frac{\mathrm{d} \varepsilon_{x}}{\mathrm{~d} z^{\prime}}+\widetilde{D^{r}} \frac{\mathrm{d} \varepsilon_{y}}{\mathrm{~d} z^{\prime}}\right] \mathrm{d} z^{\prime}\right\} } \\
&+\sum_{r}\left\{L^{r} \int_{0}^{z} \mathrm{e}^{-\lambda_{2}^{r}\left(z-z^{\prime}\right)}\left[\widetilde{E}^{r} \frac{\mathrm{d} \varepsilon_{x}}{\mathrm{~d} z^{\prime}}+\widetilde{F}^{r} \frac{\mathrm{d} \varepsilon_{y}}{\mathrm{~d} z^{\prime}}\right] \mathrm{d} z^{\prime}\right\} \\
& \sigma_{x y}=\sum_{r}\left\{D^{r} \int_{0}^{z} \mathrm{e}^{-R^{r}\left(z-z^{\prime}\right)} \frac{\mathrm{d} \varepsilon_{x y}}{\mathrm{~d} z^{\prime}} \mathrm{d} z^{\prime}\right\}
\end{aligned}
\end{aligned}
$$

where

$$
\begin{aligned}
& Y_{1}=A-\sum_{r} M^{r} \tilde{C}^{r}-\sum_{r} N^{r} \tilde{E}^{r} \\
& Y_{2}=C-\sum_{r} M^{r} \widetilde{D}^{r}-\sum_{r} N^{r} \tilde{F}^{r} \\
& Y_{3}=B-\sum_{r} K^{r} \widetilde{D}^{r}-\sum_{r} L^{r} \tilde{F}^{r} \\
& Y_{4}=C-\sum_{r} K^{r} \widetilde{C}^{r}-\sum_{r} L^{r} \tilde{E}^{r} \\
& A=\sum_{r} A^{r}, \quad B=\sum_{r} B^{r}, \quad C=\sum_{r} C^{r} \\
& M^{r}=\frac{1}{\lambda_{1}^{r}}\left[A^{r} U^{r}+C^{r}\left(-P^{r}+\lambda_{1}^{r}\right)\right]
\end{aligned}
$$




$$
\begin{aligned}
& N^{r}=\frac{1}{\lambda_{2}^{r}}\left[A^{r} U^{r}+C^{r}\left(-P^{r}+\lambda_{2}^{r}\right)\right] \\
& K^{r}=\frac{1}{\lambda_{1}^{r}}\left[C^{r} U^{r}+B^{r}\left(-P^{r}+\lambda_{1}^{r}\right)\right] \\
& L^{r}=\frac{1}{\lambda_{2}^{r}}\left[C^{r} U^{r}+B^{r}\left(-P^{r}+\lambda_{2}^{r}\right)\right]
\end{aligned}
$$

Note that all quantities given in (15) are constants. A special case of (14) is used to derive the following equations

$$
\begin{aligned}
& \sigma_{x}=K^{-3 / 2} \int_{0}^{z} G\left(z-z^{\prime}\right)\left[(H+F) \frac{\mathrm{d} \varepsilon_{x}}{\mathrm{~d} z^{\prime}}+H \frac{\mathrm{d} \varepsilon_{y}}{\mathrm{~d} z^{\prime}}\right] \mathrm{d} z^{\prime} \\
& \sigma_{y}=K^{-3 / 2} \int_{0}^{z} G\left(z-z^{\prime}\right)\left[(G+H) \frac{\mathrm{d} \varepsilon_{y}}{\mathrm{~d} z^{\prime}}+H \frac{\mathrm{d} \varepsilon_{x}}{\mathrm{~d} z^{\prime}}\right] \mathrm{d} z^{\prime} \\
& \sigma_{x y}=K^{-3 / 2} \int_{0}^{z} G_{x y}\left(z-z^{\prime}\right)\left[\frac{M}{N} \frac{\mathrm{d} \varepsilon_{x y}}{\mathrm{~d} z^{\prime}}\right] \mathrm{d} z^{\prime}
\end{aligned}
$$

where

$$
\begin{aligned}
& G(z)=\sum_{r} G^{r} \mathrm{e}^{-\lambda^{r} z} \quad \text { with } G(0)=\sum_{r} G^{r}=1 \\
& G_{x y}(z)=\sum_{r} G_{x y}^{r} \mathrm{e}^{-\lambda_{x y}^{r} z} \quad \text { with } G_{x y}(0)=\sum_{r} G_{x y}^{r}=1 \\
& K=\frac{2}{3}(F+G+H) \quad \text { and } \quad M=(H+F)(G+H)-H^{2}
\end{aligned}
$$

Constitutive equations (16) are suitable for use in sheet metals. These are expressions for the stress components in terms of the histories of total strain components $\varepsilon_{x}, \varepsilon_{y}$ and $\varepsilon_{x y}$. In the equations, $F$, $G, H, G^{r}, G_{x y}^{r}, \lambda^{r}$ and $\lambda_{x y}^{r}$ are constants with $r=1, \ldots, n$. The derivation of (16) and (17) is given in Appendix A.

It is now desirable to express the stress in terms of the histories of plastic strain components $\varepsilon_{x}^{p}$, $\varepsilon_{y}^{p}$ and $\varepsilon_{x y}^{p}$ so that

$$
\begin{aligned}
& \sigma_{x}=K^{-3 / 2} \int_{0}^{z} \rho\left(z-z^{\prime}\right)\left[(H+F) \frac{\mathrm{d} \varepsilon_{x}^{p}}{\mathrm{~d} z^{\prime}}+H \frac{\mathrm{d} \varepsilon_{y}^{p}}{\mathrm{~d} z^{\prime}}\right] \mathrm{d} z^{\prime} \\
& \sigma_{y}=K^{-3 / 2} \int_{0}^{z} \rho\left(z-z^{\prime}\right)\left[(G+H) \frac{\mathrm{d} \varepsilon_{y}^{p}}{\mathrm{~d} z^{\prime}}+H \frac{\mathrm{d} \varepsilon_{x}^{p}}{\mathrm{~d} z^{\prime}}\right] \mathrm{d} z^{\prime} \\
& \sigma_{x y}=K^{-3 / 2} \int_{0}^{z} \rho_{x y}\left(z-z^{\prime}\right)\left[\frac{M}{N} \frac{\mathrm{d} \varepsilon_{x}^{p}}{\mathrm{~d} z^{\prime}}\right] \mathrm{d} z^{\prime}
\end{aligned}
$$


The forms of expressions in the square brackets on the right hand side of (16) and (18) are assumed to be the same. These forms are established based on plastic deformation which is further discussed in Appendix B. The coefficients $F, G, H$ and $N$ involved are those of yield function (2). The kernel functions $\rho(z)$ and $\rho_{x y}(z)$ can be determined from the knowledge of $G(z)$ and $G_{x y}(z)$ using the method of Laplace transformation. Denoting the Laplace transformation of a quantity by an overhead bar for simplicity, i.e., $\bar{\sigma}=L\{\sigma\}$, etc., $(16 \mathrm{a}, \mathrm{b}, \mathrm{c})$ may be transformed into

$$
\begin{aligned}
\bar{\sigma}_{x} & =K^{-3 / 2} \bar{G}\left[(H+F) p \bar{\varepsilon}_{x}+H p \bar{\varepsilon}_{y}\right] \\
\bar{\sigma}_{y} & =K^{-3 / 2} \bar{G}\left[(G+H) p \bar{\varepsilon}_{y}+H p \bar{\varepsilon}_{x}\right] \\
\bar{\sigma}_{x y} & =\frac{M}{K^{3 / 2} N} \bar{G}_{x y} p \bar{\varepsilon}_{x y}
\end{aligned}
$$

with the initial values $\varepsilon_{x}(0)=\varepsilon_{y}(0)=\varepsilon_{x y}(0)=0$. The parameter of Laplace transformation is denoted by $p$. Similarly, $(18 \mathrm{a}, \mathrm{b}, \mathrm{c})$ are transformed into

$$
\begin{aligned}
\bar{\sigma}_{x} & =K^{-3 / 2} \bar{\rho}\left[(H+F) p \bar{\varepsilon}_{x}^{p}+H p \bar{\varepsilon}_{y}^{p}\right] \\
\bar{\sigma}_{y} & =K^{-3 / 2} \bar{\rho}\left[(G+H) p \bar{\varepsilon}_{y}^{p}+H p \bar{\varepsilon}_{x}^{p}\right] \\
\bar{\sigma}_{x y} & =\frac{M}{K^{3 / 2} N} \bar{\rho}_{x y} p \bar{\varepsilon}_{x y}^{p}
\end{aligned}
$$

with $\bar{\varepsilon}_{x}^{p}(0)=\bar{\varepsilon}_{y}^{p}(0)=\bar{\varepsilon}_{x y}^{p}(0)=0$. The plastic strain components in (20) are now expressed by the difference between the total strain and the elastic strain. Since the expressions in the brackets of (16) and (18) have been obtained from the consideration of plastic deformation, the elastic behavior derived from (16) is not exact and it may be obtained from (16) by setting $z \rightarrow 0$. A further approximation is made to retain only terms of elastic strain in the direction of the applied load so that for loading in the $x$-direction, the plastic strains are

$$
\varepsilon_{x}^{p}=\varepsilon_{x}-\varepsilon_{x}^{e}=\varepsilon_{x}-\frac{\sigma_{x} K^{3 / 2}}{H+F} \quad \text { and } \quad \varepsilon_{y}^{p}=\varepsilon_{y}-\varepsilon_{y}^{e} \approx \varepsilon_{y}
$$

and for loading in the $y$-direction, the plastic strains are

$$
\varepsilon_{x}^{p} \approx \varepsilon_{x} \quad \text { and } \quad \varepsilon_{y}^{p}=\varepsilon_{y}-\frac{\sigma_{y} K^{3 / 2}}{G+H}
$$

The shear component is

$$
\varepsilon_{x y}^{p}=\varepsilon_{x y}-\frac{\sigma_{x y} K^{3 / 2} N}{M}
$$

Substitute (22a) into (20a), (22b) into (20b) and (22c) into (20c). The resulting equations and (19) are then combined, respectively, to yield the following expressions

$$
\bar{\rho}=\frac{\bar{G}}{1-p \bar{G}}
$$




$$
\bar{\rho}_{x y}=\frac{\bar{G}_{x y}}{1-p \bar{G}_{x y}}
$$

Using (23) and after the inverse Laplace transformation, it may be shown, following Valanis (1980) and Wu and Yang (1983), that the kernel functions are

$$
\begin{aligned}
& \rho(z)=\delta(z)+\rho_{1}(z) \\
& \rho_{x y}(z)=\delta(z)+\rho_{x y 1}(z)
\end{aligned}
$$

where $\delta(z)$ is the Dirac delta function; and $\rho_{1}(z)$ and $\rho_{x y 1}(z)$ are given by

$$
\begin{aligned}
& \rho_{1}(z)=\sum_{r=1}^{n-1} R^{r} \mathrm{e}^{-\alpha_{r} z} \\
& \rho_{x y 1}(z)=\sum_{r=1}^{n-1} R_{x y}^{r} \mathrm{e}^{-\alpha_{x y r} z}
\end{aligned}
$$

where $R^{r}, R_{x y}^{r}, \alpha_{r}$ and $\alpha_{x y r}$ are positive constants. Substituting (24) into (18), the constitutive equations for sheet metals may be obtained as

$$
\begin{aligned}
& \sigma_{x}=K^{-3 / 2}\left[(H+F) \frac{\mathrm{d} \varepsilon_{x}^{p}}{\mathrm{~d} z}+H \frac{\mathrm{d} \varepsilon_{y}^{p}}{\mathrm{~d} z}\right]+K^{-3 / 2} \int_{0}^{z} \rho_{1}\left(z-z^{\prime}\right)\left[(H+F) \frac{\mathrm{d} \varepsilon_{x}^{p}}{\mathrm{~d} z^{\prime}}+H \frac{\mathrm{d} \varepsilon_{y}^{p}}{\mathrm{~d} z^{\prime}}\right] \mathrm{d} z^{\prime} \\
& \sigma_{y}=K^{-3 / 2}\left[(G+H) \frac{\mathrm{d} \varepsilon_{y}^{p}}{\mathrm{~d} z}+H \frac{\mathrm{d} \varepsilon_{x}^{p}}{\mathrm{~d} z}\right]+K^{-3 / 2} \int_{0}^{z} \rho_{1}\left(z-z^{\prime}\right)\left[(G+H) \frac{\mathrm{d} \varepsilon_{y}^{p}}{\mathrm{~d} z^{\prime}}+H \frac{\mathrm{d} \varepsilon_{x}^{p}}{\mathrm{~d} z^{\prime}}\right] \mathrm{d} z^{\prime}
\end{aligned}
$$

and

$$
\sigma_{x y}=K^{-3 / 2}\left[\frac{M}{N} \frac{\mathrm{d} \varepsilon_{x y}}{\mathrm{~d} z}\right]+K^{-3 / 2} \int_{0}^{z} \rho_{x y 1}\left(z-z^{\prime}\right)\left[\frac{M}{N} \frac{\mathrm{d} \varepsilon_{x y}}{\mathrm{~d} z^{\prime}}\right] \mathrm{d} z^{\prime}
$$

Equations (26) are the endochronic constitution equations for sheet metals expressed in terms of the histories of plastic strain. These equations are valid in the plastic region only. In order that the strain-hardening may be discussed, the intrinsic time $z$ is further scaled by introducing another intrinsic time $\zeta$ through the relation

$$
\mathrm{d} z=\frac{\mathrm{d} \zeta}{f(\zeta)}
$$

with initial conditions $z=0$ and $\mathrm{d} z / \mathrm{d} \zeta=1$ at $\zeta=0$. In (27), $f(\zeta)$ is a scaling function and will be identified later with isotropic hardening. Thus, $f(\zeta)$ may be referred to as the isotropic hardening function. When $z=0$, no plastic strain has as yet occurred and at this state $\zeta=0$ and $f(0)=1$. For $z>0^{+}$, eqns (26) apply.

Denoting

$$
r_{x}=K^{-3 / 2} \int_{0}^{z} \rho_{1}\left(z-z^{\prime}\right)\left[(H+F) \frac{\mathrm{d} \varepsilon_{x}^{p}}{\mathrm{~d} z^{\prime}}+H \frac{\mathrm{d} \varepsilon_{y}^{p}}{\mathrm{~d} z^{\prime}}\right] \mathrm{d} z^{\prime}
$$




$$
\begin{aligned}
& r_{y}=K^{-3 / 2} \int_{0}^{z} \rho_{1}\left(z-z^{\prime}\right)\left[(G+H) \frac{\mathrm{d} \varepsilon_{y}^{p}}{\mathrm{~d} z^{\prime}}+H \frac{\mathrm{d} \varepsilon_{x}^{p}}{\mathrm{~d} z^{\prime}}\right] \mathrm{d} z^{\prime} \\
& r_{x y}=K^{-3 / 2} \int_{0}^{z} \rho_{x y 1}\left(z-z^{\prime}\right)\left[\frac{M}{N} \frac{\mathrm{d} \varepsilon_{x y}}{\mathrm{~d} z^{\prime}}\right] \mathrm{d} z^{\prime}
\end{aligned}
$$

eqns (26a) and (26b) may be further written as

$$
\begin{aligned}
& {\left[(H+F) \frac{\mathrm{d} \varepsilon_{x}^{p}}{\mathrm{~d} \zeta}+H \frac{\mathrm{d} \varepsilon_{y}^{p}}{\mathrm{~d} \zeta}\right]=\frac{\sigma_{x}-r_{x}}{K^{-3 / 2} f}} \\
& {\left[(G+H) \frac{\mathrm{d} \varepsilon_{y}^{p}}{\mathrm{~d} \zeta}+H \frac{\mathrm{d} \varepsilon_{x}^{p}}{\mathrm{~d} \zeta}\right]=\frac{\sigma_{y}-r_{y}}{K^{-3 / 2} f}}
\end{aligned}
$$

which may then be solved for

$$
M \frac{\mathrm{d} \varepsilon_{x}^{p}}{\mathrm{~d} \zeta}=\frac{(G+H)\left(\sigma_{x}-r_{x}\right)}{K^{-3 / 2} f}-\frac{H\left(\sigma_{y}-r_{y}\right)}{K^{-3 / 2} f}
$$

and

$$
M \frac{\mathrm{d} \varepsilon_{y}^{p}}{\mathrm{~d} \zeta}=\frac{(H+F)\left(\sigma_{y}-r_{y}\right)}{K^{-3 / 2} f}-\frac{H\left(\sigma_{x}-r_{y}\right)}{K^{-3 / 2} f}
$$

Also, (26c) is rewritten as

$$
\frac{M}{N} \frac{\mathrm{d} \varepsilon_{x y}^{p}}{\mathrm{~d} \zeta}=\frac{\sigma_{x y}-r_{x y}}{K^{-3 / 2} f}
$$

Therefore, in an anisotropic sheet, it takes a multiaxial stress state to produce a single plastic strain component. Equations $(30 \mathrm{a}, \mathrm{b}, \mathrm{c})$ may be considered as the 'flow rule' using concept of the flow theory of plasticity. Comparing (30) with (B2), it is seen that the plastic strain increments for the two cases are along the same direction, i.e., the normality condition is satisfied. Finally, the plastic incompressibility is assumed so that

$$
\mathrm{d} \varepsilon_{x}^{p}+\mathrm{d} \varepsilon_{y}^{p}+\mathrm{d} \varepsilon_{z}^{p}=0
$$

\section{The definition of intrinsic time and the yield function}

It was shown by $\mathrm{Wu}$ et al. (1995a) that the form of the yield function in the endochronic theory depends closely on the definition of intrinsic time. For sheet metals, it is shown in this section that the proposed definition of intrinsic time leads to Hill's 1948 quadratic yield criterion. The intrinsic time is defined using the concept of equivalent plastic strain increment discussed in Appendix B. Thus, 


$$
\begin{array}{r}
\mathrm{d} \zeta^{2}=\left\{\frac{G+H}{K^{3}}\left[(H+F) \frac{\mathrm{d} \varepsilon_{x}^{p}}{\mathrm{~d} \zeta}+H \frac{\mathrm{d} \varepsilon_{y}^{p}}{\mathrm{~d} \zeta}\right]^{2}-\frac{2 H}{K^{3}}\left[(H+F) \frac{\mathrm{d} \varepsilon_{x}^{p}}{\mathrm{~d} \zeta}+H \frac{\mathrm{d} \varepsilon_{y}^{p}}{\mathrm{~d} \zeta}\right]\left[(G+H) \frac{\mathrm{d} \varepsilon_{y}^{p}}{\mathrm{~d} \zeta}+H \frac{\mathrm{d} \varepsilon_{x}^{p}}{\mathrm{~d} \zeta}\right]\right. \\
\left.+\frac{H+F}{K^{3}}\left[(G+H) \frac{\mathrm{d} \varepsilon_{y}^{p}}{\mathrm{~d} \zeta}+H \frac{\mathrm{d} \varepsilon_{x}^{p}}{\mathrm{~d} \zeta}\right]^{2}+\frac{2 N}{K^{3}}\left(\frac{M}{N} \frac{\mathrm{d} \varepsilon_{x y}^{p}}{\mathrm{~d} \zeta}\right)^{2}\right\} \mathrm{d} \zeta^{2}
\end{array}
$$

Upon the use of (29a), (29b) and (30c), (32) reduces to

$$
\begin{aligned}
\left\{\frac{(G+H)}{K^{3}}\left(\frac{\sigma_{x}-r_{x}}{K^{-3 / 2} f}\right)^{2}-\frac{2 H}{K^{3}}\left(\frac{\sigma_{x}-r_{x}}{K^{-3 / 2} f}\right)\right. & \left(\frac{\sigma_{y}-r_{y}}{K^{-3 / 2} f}\right) \\
& \left.+\frac{(H+F)}{K^{3}}\left(\frac{\sigma_{y}-r_{y}}{K^{-3 / 2} f}\right)^{2}+\frac{2 N}{K^{3}}\left(\frac{\sigma_{x y}-r_{x y}}{K^{-3 / 2} f}\right)^{2}-1\right\} \mathrm{d} \zeta^{2}=0
\end{aligned}
$$

Thus, either $\mathrm{d} \zeta=0$ and the quantity in the bracket \{\}$\neq 0$, or $\mathrm{d} \zeta \neq 0$ and the bracket \{\}$=0$. The case of $\mathrm{d} \zeta=0$ corresponds to the elastic behavior and the case of $\mathrm{d} \zeta \neq 0$ corresponds to the plastic behavior. In the latter case, after simplification, one obtains

$$
(G+H)\left(\sigma_{x}-r_{x}\right)^{2}-2 H\left(\sigma_{x}-r_{x}\right)\left(\sigma_{y}-r_{y}\right)+(H+F)\left(\sigma_{y}-r_{y}\right)^{2}+2 N\left(\sigma_{x y}-r_{x y}\right)^{2}=f^{2}
$$

This is exactly the same as (2) and is an extension of the yield criterion for sheet metals proposed by Hill in 1948. This equation also identifies $f$ as the isotropic hardening function, because it specifies the size of the yield surface. It also shows that $r_{x}, r_{y}$ and $r_{x y}$ specify the center of the yield surface and therefore, represent the kinematic hardening. These are also known as the components of the back stress.

The coefficients $F, G, H$ and $N$ are determined from experiments. Several tests have been used in the literature for this purpose. They range from tests that determine yield stresses to those that determine the width to thickness plastic strain ratio $R$. The latter tests do not have the same degree of accuracy, however and they involve assumptions and other factors. Thus, the values of the coefficients determined entirely from the yield-stress tests are different than those determined from the plastic strain ratio tests or those determined from the mixture of the two types of tests. This, of course, is not acceptable, because $F, G, H$ and $N$ are material constants and for a given metal sheet, their values are fixed.

Of the two types of tests, the yield-stress tests are the simpler tests. Although the yield has several definitions, i.e., the proportional limit, proof strain, or backward extrapolation, as long as the definition is chosen, a rather well-defined yield stress can be determined from the experiments and the result is rather repeatable although subjected to some degree of expected experimental scatter. On the other hand, there are many factors of uncertainty associated with the plastic strain ratio test. Experimentally, the measured value in the thickness strain does not have the same degree of accuracy as in the width and longitudinal strains in a sheet metal due to its thinness. Therefore, an experimentally determined ratio between the width and thickness strain is not of high degree of accuracy. Experiments show that the $R$-ratio varies with strain. According to Mellor (1982) for titanium 115 , the $R$-ratio varies greatly with the increasing plastic strain if it is defined as the ratio of the width to thickness strain. The ratio varies the most at the transition zone from the elastic to plastic strain. But if the $R$-ratio is defined as the ratio of plastic strain increments, then it almost 
remains constant over the whole plastic strain range tested, up to a strain of $13 \%$. However, the experimental results are not sufficiently accurate to allow computation of the ratio of plastic strain increments closer to the initial yielding zone. The curve for $R$ rises slightly with decreasing strain, but an extrapolation of this curve to zero strain is not recommended. Experiments were also conducted by Lin and Ding (1995) for as received and cold-rolled aluminum sheets using cruciform plate specimen. A similar conclusion was also obtained regarding the $R$-ratio at small plastic strain. The authors stated that $R$ could not be reasonably determined when the plastic strain is infinitesimal due to the quite severe scattering of the plastic strain increments. Finally, it should be pointed out that the method of determining the coefficients $F, G, H$ and $N$ by use of the $R$-ratios does not conform to the traditional method of plasticity. Traditionally, the yield function is determined from the yield stresses and not from the flow rule. Since $R$ is determined by the flow rule, the determination of the yield function in terms of $R$ would have the same effect as in terms of the flow rule. Due to the aforementioned reasons, it is believed that the coefficients of the yield function should be determined from tests that determine the yield-stresses and will be subsequently discussed.

These coefficients may be determined at the condition of initial yielding. Thus, $r_{x}=r_{y}=r_{x y}=0$ and $f=1$ and (34) reduces to

$$
(G+H) \sigma_{x}^{2}-2 H \sigma_{x} \sigma_{y}+(H+F) \sigma_{y}^{2}+2 N \sigma_{x y}^{2}=1
$$

Note that $x$ denotes the rolling direction and $y$ the transverse direction. In a tension test along the $x$-direction, the stresses are $\sigma_{x} \neq 0, \sigma_{y}=\sigma_{x y}=0$ and (35) reduces to

$$
G+H=\left(\sigma_{x}^{Y}\right)^{-2}
$$

Similarly, a tension test along the $y$-direction leads to

$$
H+F=\left(\sigma_{y}^{Y}\right)^{-2}
$$

Yielding under equibiaxial tension occurs when $\sigma_{x}=\sigma_{y}=\sigma_{B}^{Y}$. In this case, (35) reduces to

$$
G+F=\left(\sigma_{B}^{Y}\right)^{-2}
$$

Due to the usual assumption that hydrostatic stress does not affect yielding, $\sigma_{B}^{Y}$ is also the compressive yield stress perpendicular to the sheet. The through-thickness compression test was carried out by Naruse et al. (1992). Cylindrical specimens were prepared from discs of each sheet material glued together with an epoxy adhesive. The specimens were tested in compression using Teflon sheet and graphite grease for lubrication between the specimen ends and platen of the test machine. Factors of uncertainty arose from the epoxy adhesive, the Teflon sheet and graphite grease. Therefore, the equibiaxial tension test is preferred over the aforementioned compression test. Equations $(36 \mathrm{a}, \mathrm{b}, \mathrm{c})$ can be solved for $G, F$ and $H$ to yield

$$
\begin{aligned}
& 2 G=\frac{1}{\left(\sigma_{x}^{Y}\right)^{2}}-\frac{1}{\left(\sigma_{y}^{Y}\right)^{2}}+\frac{1}{\left(\sigma_{B}^{Y}\right)^{2}} \\
& 2 F=\frac{1}{\left(\sigma_{y}^{Y}\right)^{2}}-\frac{1}{\left(\sigma_{x}^{Y}\right)^{2}}+\frac{1}{\left(\sigma_{B}^{Y}\right)^{2}}
\end{aligned}
$$




$$
2 H=\frac{1}{\left(\sigma_{x}^{Y}\right)^{2}}+\frac{1}{\left(\sigma_{y}^{Y}\right)^{2}}-\frac{1}{\left(\sigma_{B}^{Y}\right)^{2}}
$$

Finally, the coefficient $N$ may be determined from the tension test of a specimen cut at $45^{\circ}$ angle with the $x$-direction. The tensile yield stress for this specimen is denoted by $\sigma_{45}$ and $\sigma_{x}=\sigma_{y}=\sigma_{x y}=\frac{1}{2} \sigma_{45}$. Using this condition, it may be found from eqn (35) that

$$
2 N=\left(\frac{\sigma_{45}}{2}\right)^{-2}-\left(\sigma_{B}^{Y}\right)^{-2}
$$

Since $\sigma_{45}$ is easily determined experimentally, $N$ is thus determined from (37d). The shear yield stress $\sigma_{x y}^{Y}$ is determined in pure shear with material element parallel to the orthotropic axes. It may be shown from (35) that

$$
\sigma_{x y}^{Y}=\frac{1}{\sqrt{2 N}}
$$

However, in sheet metals, pure shear is difficult to realize experimentally. Also, the simple shear test is sometimes used in the literature to determine $N$. The stress state of this test is never simple, however and the test can at best be used as an approximation. It has thus been shown that the coefficients of the yield function can be determined by a set of tension tests. Well-controlled tension tests are simple to perform. The equibiaxial test can be carried out by use of cruciform specimens as in Makinde et al. (1992) and Lin and Ding (1995).

In summary, using this formulation, the initial material anisotropy is specified by material constants $(F, G, H, N)$. The strain hardening is specified by an isotropic hardening function $f$ and the kernel functions $\rho(z)$ and $\rho_{x y}(z)$ which characterize kinematic hardening. The kinematic hardening describes the deformation induced anisotropy. The constitutive equations are given in (26) with the back stress expressed by (28). From (26), the yield function and flow rule are derived and given in (34) and (30), respectively. Finally, the intrinsic time is defined by (32).

\section{Material parameters of the endochronic theory}

The constitutive equations of the theory are $(26 a, b, c)$ with the intrinsic time defined by (32). In these equations, $F, G, H, N, K$ and $M$ are known as described in the previous section. The determination of parameters associated with kernel functions $\rho_{1}(z)$ and $\rho_{x y 1}(z)$ need to be discussed. In the first place, the yield stresses will be identified by setting $z=0^{+}$in (26). In the case of uniaxial stress $\sigma_{x}$ loaded from the initial state, $\left(\sigma_{y}-r_{y}\right)$ is zero so that, from (29b),

$$
(G+H) \frac{\mathrm{d} \varepsilon_{y}^{p}}{\mathrm{~d} \zeta}+H \frac{\mathrm{d} \varepsilon_{x}^{p}}{\mathrm{~d} \zeta}=0
$$

This equation is then substituted into (32) to find, for the uniaxial stress in the $x$-direction, that 


$$
\mathrm{d} \zeta^{2}=\frac{G+H}{K^{3}}\left[(H+F) \frac{\mathrm{d} \varepsilon_{y}^{p}}{\mathrm{~d} \zeta}+H \frac{\mathrm{d} \varepsilon_{x}^{p}}{\mathrm{~d} \zeta}\right]^{2} \mathrm{~d} \zeta^{2}
$$

or

$$
\left|(H+F) \frac{\mathrm{d} \varepsilon_{y}^{p}}{\mathrm{~d} \zeta}+H \frac{\mathrm{d} \varepsilon_{x}^{p}}{\mathrm{~d} \zeta}\right|=\frac{K^{3 / 2}}{(G+H)^{1 / 2}}
$$

Therefore, when $z=0^{+}$, the isotropic hardening function $f_{z=0}=1$, and by the use of (41) and (36a), (26a) reduces to

$$
\sigma_{x}=\sigma_{x}^{Y}
$$

Similarly, in the case of uniaxial stress $\sigma_{y}$, when $z=0^{+},(26 \mathrm{~b})$ reduces to

$$
\sigma_{y}=\sigma_{y}^{Y}
$$

and in the case of pure shear, when $z=0^{+},(26 \mathrm{c})$ reduces to

$$
\sigma_{x y}=\sigma_{x y}^{Y}
$$

The parameters of the model can be determined by considering the plane strain extension condition, where $\mathrm{d} \varepsilon_{x}^{p} \neq 0$ but $\mathrm{d} \varepsilon_{y}^{p}=0$ and $\mathrm{d} \varepsilon_{x y}^{p}=0$. In this case, (26a) reduces to

$$
\sigma_{x}=K^{-3 / 2}\left[(H+F) \frac{\mathrm{d} \varepsilon_{x}^{p}}{\mathrm{~d} z}\right]+K^{-3 / 2} \int_{0}^{z} \rho_{1}\left(z-z^{\prime}\right)\left[(H+F) \frac{\mathrm{d} \varepsilon_{x}^{p}}{\mathrm{~d} z^{\prime}}\right] \mathrm{d} z^{\prime}
$$

The general form of kernel function $\rho_{1}(z)$ is given in (25). It has been found in previous applications that only one exponential term will capture the essential feature of metal behavior. The following form is, therefore, used in the subsequent discussion:

$$
\rho_{1}(z)=R_{1} \mathrm{e}^{-\alpha z}
$$

Using (32), the intrinsic time for the plane strain extension is

$$
\mathrm{d} \zeta= \pm\left((H+F) M K^{-3}\right)^{1 / 2} \mathrm{~d} \varepsilon_{x}^{p}
$$

so that

$$
\frac{\mathrm{d} \varepsilon_{x}^{p}}{\mathrm{~d} \zeta}= \pm\left(\frac{K^{3}}{(H+F) M}\right)^{1 / 2}
$$

where the ' + ' sign corresponds to loading and ' - ' corresponds to unloading. If the isotropic strain hardening function is given by the linear form as

$$
\frac{\mathrm{d} \zeta}{\mathrm{d} z}=f=1+\beta \zeta
$$

where $\beta$ is a parameter that describes isotropic hardening, then, in the case of loading, (42) reduces to the following expression by use of (43), (45) and (46): 


$$
\sigma_{x}=\left(\frac{H+F}{M}\right)^{1 / 2}\left\{(1+\beta \zeta)+\frac{R_{1}}{(n+1) \beta}\left[(1+\beta \zeta)-\frac{1}{(1+\beta \zeta)^{n}}\right]\right\}
$$

This equation describes the stress-plastic strain curve. In the equation,

$$
n=\alpha / \beta
$$

The yield point $\sigma_{p l \cdot \varepsilon \cdot x}^{y}$ of this curve may be found by setting $\zeta \rightarrow 0$. Thus,

$$
\sigma_{p l \cdot \varepsilon \cdot x}^{y}=\left(\frac{H+F}{M}\right)^{1 / 2}
$$

On the other hand, the asymptote of the curve is obtained by setting $\zeta \rightarrow \infty$ in (47) and is given by

$$
\sigma_{x}=\left(\frac{H+F}{M}\right)^{1 / 2}\left(1+\frac{R_{1}}{(n+1) \beta}\right)(1+\beta \zeta)
$$

The intercept $\sigma_{x}^{0}$ of the asymptote with the stress axis is obtained by setting $\zeta=0$ in (50). Thus,

$$
\sigma_{x}^{0}=\left(\frac{H+F}{M}\right)^{1 / 2}\left(1+\frac{R_{1}}{(n+1) \beta}\right)
$$

Finally, the slope of the asymptote (50), is, by use of (49) and (51)

$$
E^{T}=\frac{\mathrm{d} \sigma_{x}}{\mathrm{~d} \varepsilon_{x}^{p}}=\frac{\mathrm{d} \sigma_{x}}{\mathrm{~d} \zeta} \frac{\mathrm{d} \sigma}{\mathrm{d} \varepsilon_{x}^{p}}=\left((H+F) M K^{-3}\right)^{1 / 2} \beta \sigma_{x}^{0}
$$

Equation (47) may be simplified by observing that, from (44) and (52) and in the case of loading, the following relations hold

$$
\beta \zeta=\left(\frac{E^{T}}{\sigma_{x}^{0}}\right) \varepsilon_{x}^{p}=m \varepsilon_{x}^{p} \quad \text { with } m=\left(\frac{E^{T}}{\sigma_{x}^{0}}\right)
$$

Therefore, by use of (49), (51) and (53), (47) may be rewritten as

$$
\sigma_{x}=\sigma_{p l \cdot \varepsilon \cdot x}^{y}\left\{\left(1+m \varepsilon_{x}^{p}\right)+\left(\frac{\sigma_{x}^{0}}{\sigma_{p l \cdot \varepsilon \cdot x}^{y}}-1\right)\left[\left(1+m \varepsilon_{x}^{p}\right)-\frac{1}{\left(1+m \varepsilon_{x}^{p}\right)^{n}}\right]\right\}
$$

where

$$
\begin{aligned}
\text { isotropic hardening } & =\sigma_{p l \cdot \varepsilon \cdot x}^{y}\left(1+m \varepsilon_{x}^{p}\right) \\
\text { kinematic hardening } & =r_{x}=\left(\sigma_{x}^{0}-\sigma_{p l \cdot \varepsilon \cdot x}^{y}\right)\left[\left(1+m \varepsilon_{x}^{p}\right)-\frac{1}{\left(1+m \varepsilon_{x}^{p}\right)^{n}}\right]
\end{aligned}
$$

If an experimental stress-plastic strain curve is available for the plane strain extension case, then $\sigma_{p l \cdot \varepsilon x}^{y}, \sigma_{x}^{0}$ and $E^{T}$ can be experimentally determined and $m$ is known from (53). An optimization procedure may be used to determine parameter $n$ by requiring the calculated curve to pass through 
certain experimental points on the graph. The optimization procedure for the endochronic theory was discussed by Jao et al. (1991).

Plane strain extension is not easily accomplished experimentally. Some published works are Wagoner and Wang (1979), Wagoner (1980) and Taha et al. (1995). In Table 1 of Wagoner (1980), the saturation model gives the experimental effective stress vs effective strain relation. This relation is transformed into an axial stress vs axial strain relation by use of the equations in Appendix C of that paper. The experimental curve for extension along the rolling direction for 2036-T4 aluminum alloy is plotted in Fig. 1. In the data conversion, the plastic anisotropy parameter $r$ is taken as 0.7 according to Fig. 3 of that paper. Figure 1 shows the theoretical plane strain stressstrain curve by use of (54). The evolution of back stress is also shown and it has been calculated by use of (55). The following parameters were used in the calculation: $\sigma_{p l \cdot \varepsilon x}^{y}=260 \mathrm{MPa}$, $\sigma_{x}^{0}=360 \mathrm{MPa}, E^{T}=648 \mathrm{MPa}, m=1.8$ and $n=12$.

Most equibiaxial tests found in the literature are stress-controlled. In that case, $\sigma_{x}=\sigma_{y}=\sigma$ and $\varepsilon_{x}^{p} \neq \varepsilon_{y}^{p}$. On the other hand, in a strain-controlled test, it is possible to perform an experiment so that $\varepsilon_{x}^{p}=\varepsilon_{y}^{p}=\varepsilon^{p}$, but $\sigma_{x} \neq \sigma_{y}$. The latter test is suitable to the present theory. However, no experimental result has been found in the literature. In the strain-controlled equibiaxial extension test, the intrinsic time is from (32) given by

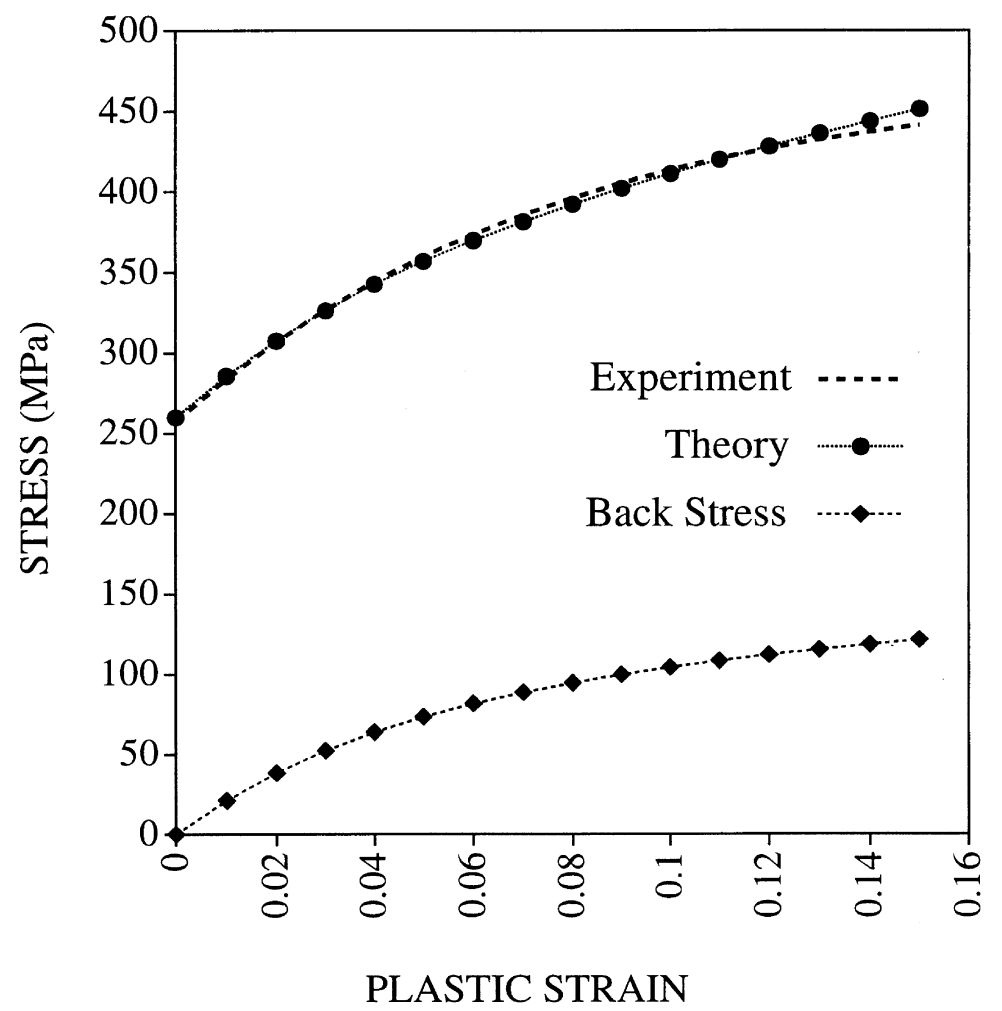

Fig. 1. Stress-plastic strain curve for plane strain extension. 


$$
\mathrm{d} \zeta= \pm\left((4 H+F+G) M K^{-3}\right)^{1 / 2} \mathrm{~d} \varepsilon_{x}^{p}
$$

Using the kernel function (43), (26a) reduces to

$$
\sigma_{x}=\sigma_{x \cdot b i}^{v}\left\{\left(1+m^{\prime} \varepsilon^{p}\right)+\left(\frac{\sigma_{x \cdot b i}^{0}}{\sigma_{x \cdot b i}^{y}}-1\right)\left[\left(1+m^{\prime} \varepsilon^{p}\right)-\frac{1}{\left(1+m^{\prime} \varepsilon^{p}\right)^{n}}\right]\right\}
$$

where the yield stress is

$$
\sigma_{x \cdot b i}^{y}=\frac{2 H+F}{M^{1 / 2}(4 H+F+G)^{1 / 2}}
$$

the stress intercept $\sigma_{x \cdot b i}^{0}$ and the slope of the asymptote $E_{b i}^{T}$ are related by

$$
E_{b i}^{T}=\left((4 H+F+G) M K^{-3}\right)^{1 / 2} \beta \sigma_{x \cdot b i}^{0}
$$

and furthermore,

$$
\beta \zeta=m^{\prime} \varepsilon^{p} \quad \text { with } m^{\prime}=\left(\frac{E_{b i}^{T}}{\sigma_{x \cdot b i}^{0}}\right)
$$

eqn (57) is the stress-strain relation in the $x$-direction for equibiaxial extension. It is then easy to show from (26b) that

$$
\sigma_{y}=\left(\frac{2 H+G}{2 H+F}\right) \sigma_{x}
$$

and the yield stress in the $y$-direction is

$$
\sigma_{y \cdot b i}^{y}=\frac{2 H+G}{M^{1 / 2}(4 H+F+G)^{1 / 2}}
$$

Note that the expressions of (58) and (62) satisfy the yield criterion (35).

\section{The plastic strain ratio}

The plastic strain ratio $R$ is determined by use of the flow rule. The ratio $R_{\alpha}$ for a tension specimen cut at an angle $\alpha$ with respect to the $x$-direction is defined by

$$
R_{\alpha}=\frac{\mathrm{d} \varepsilon_{y^{\prime}}^{p}}{\mathrm{~d} \varepsilon_{z^{\prime}}^{p}}
$$

where $x^{\prime}$ is along the longitudinal direction of the specimen; $y^{\prime}$ is the transverse direction; and $z^{\prime}=z$. Note that $x^{\prime}$ is making an angle $\alpha$ with the $x$-direction. By use of coordinate transformation, (63) is transformed into 


$$
R_{\alpha}=-\frac{\mathrm{d} \varepsilon_{x}^{p} \sin ^{2} \alpha+\mathrm{d} \varepsilon_{y}^{p} \cos ^{2} \alpha-2 \mathrm{~d} \varepsilon_{x y}^{p} \sin \alpha \cos \alpha}{\mathrm{d} \varepsilon_{x}^{p}+\mathrm{d} \varepsilon_{y}^{p}}
$$

The flow rule given by $(30 \mathrm{a}, \mathrm{b}, \mathrm{c})$ is now substituted into (64) to obtain

$$
\begin{aligned}
R_{\alpha}=-\left\{\left[(G+H) \sigma_{x}-H \sigma_{y}\right]\right. & \sin ^{2} \alpha+\left[(H+F) \sigma_{y}-H \sigma_{x}\right] \cos ^{2} \alpha \\
& \left.-2 N \sigma_{x y} \sin \alpha \cos \alpha\right\} /\left\{\left[(G+H) \sigma_{x}-H \sigma_{y}\right]+\left[(H+F) \sigma_{y}-H \sigma_{x}\right]\right\}
\end{aligned}
$$

Note that the initial yielding is being considered so that $r_{x}=r_{y}=r_{x y}=0$ and $f=1$. For a tensile specimen at an angle $\alpha$ to the rolling direction,

$$
\sigma_{x}=\sigma \cos ^{2} \alpha, \quad \sigma_{y}=\sigma \sin ^{2} \alpha \quad \text { and } \quad \sigma_{x y}=\sigma \sin \alpha \cos \alpha
$$

where $\sigma$ is the tensile stress applied to the specimen. Then, (65) reduces to

$$
\begin{aligned}
R_{\alpha}=-\left\{\left[(G+H) \sigma \cos ^{2} \alpha-H \sigma \sin ^{2} \alpha\right] \sin ^{2} \alpha+\right. & {\left[(H+F) \sigma \sin ^{2} \alpha-H \sigma \cos ^{2} \alpha\right] \cos ^{2} \alpha } \\
- & \left.2 N \sigma \sin ^{2} \alpha \cos ^{2} \alpha\right\} /\left\{G \sigma \cos ^{2} \alpha+F \sigma \sin ^{2} \alpha\right\}
\end{aligned}
$$

Substituting $\alpha=0,90$ and $45^{\circ}$, respectively, into (67), the following expressions are obtained

$$
\begin{aligned}
& R_{0}=\frac{H}{G} \\
& R_{90}=\frac{H}{F} \\
& R_{45}=\frac{1}{2}\left(\frac{2 N}{G+F}-1\right)
\end{aligned}
$$

For the as-received condition, $r_{x}=r_{y}=r_{x y}=0$ and the tensile yield stress $\sigma_{\alpha}$ at any orientation $\alpha$ may be derived based on the yield function given by (35). If the tension specimen is cut at an angle $\alpha$, the stress components are given by

$$
\sigma_{x}=\sigma_{\alpha} \cos ^{2} \alpha, \quad \sigma_{y}=\sigma_{\alpha} \sin ^{2} \alpha \text { and } \sigma_{x y}=\sigma_{\alpha} \sin \alpha \cos \alpha
$$

By the substitution of (69) and (37), the yield function (35) reduces then to

$$
\sigma_{\alpha}^{2}\left[\frac{1}{\left(\sigma_{x}^{Y}\right)^{2}} \cos ^{4} \alpha+\left(\frac{1}{\left(\sigma_{x y}^{Y}\right)^{2}}-\frac{1}{\left(\sigma_{x}^{Y}\right)^{2}}-\frac{1}{\left(\sigma_{y}^{Y}\right)^{2}}+\frac{1}{\left(\sigma_{B}^{Y}\right)^{2}}\right) \cos ^{2} \alpha \sin ^{2} \alpha+\frac{1}{\left(\sigma_{y}^{Y}\right)^{2}} \sin ^{4} \alpha\right]=1
$$

To each stress state on the yield surface, denoted by $\left(\sigma_{x}, \sigma_{y}, \sigma_{x y}\right)$, there corresponds a uniaxial stress state, denoted by $\left(\sigma_{\alpha}, \alpha\right)$, also on the yield surface. By fixing $\alpha$, the uniaxial yield stress is determined from (70). Some special cases are

$$
\sigma_{0}=\sigma_{x}^{Y}, \quad \sigma_{90}=\sigma_{y}^{Y} \quad \text { and } \quad \sigma_{45}=\frac{2}{\sqrt{\frac{1}{\left(\sigma_{x y}^{Y}\right)^{2}}+\frac{1}{\left(\sigma_{B}^{Y}\right)^{2}}}}
$$

for $\alpha=0,90$ and $45^{\circ}$, respectively. Equation (70) may be plotted to obtain the distribution of $\sigma_{\alpha}$ 
with respect to $\alpha$. Note that (71) are given in terms of the yield stresses and they can be shown to be the same as those of Hill (1990).

\section{Conclusion}

The quadratic anisotropic plane-stress yield criterion and its associated normality rule of the plastic strain rate have been derived based on an endochronic theory of plasticity. The range of validity of the sheet metal plasticity with quadratic anisotropic yield function is greatly extended by incorporation of kinematic hardening into the model and it can account for the 'anomalous behavior'.

It has been proposed that the coefficients of the anisotropic quadratic yield function be determined by the yield stresses using a set of tension tests. In addition, it has been shown that the expression of intrinsic time in the endochronic theory is closely related to the form of the yield function.

\section{Appendix A}

It is shown in this Appendix that (16) and (17) describe a special case of (14). When $Y_{1}=Y_{2}=Y_{3}=Y_{4}=0$ and $\lambda_{1}^{r}=\lambda_{2}^{r}=\lambda^{r},(14 \mathrm{a})$ and (14b) may be written as

$$
\begin{aligned}
& \sigma_{x}=\sum_{r}\left[\int_{0}^{z}\left(\widetilde{C^{r}} M^{r}+\widetilde{E}^{r} N^{r}\right) \mathrm{e}^{-\lambda^{r}\left(z-z^{\prime}\right)} \frac{\mathrm{d} \varepsilon_{x}}{\mathrm{~d} z^{\prime}} \mathrm{d} z^{\prime}\right]+\sum_{r}\left[\int_{0}^{z}\left(\widetilde{D^{r}} M^{r}+\widetilde{F}^{r} N^{r}\right) \mathrm{e}^{-\lambda^{r}\left(z-z^{\prime}\right)} \frac{\mathrm{d} \varepsilon_{y}}{\mathrm{~d} z^{\prime}} \mathrm{d} z^{\prime}\right] \\
& \sigma_{y}=\sum_{r}\left[\int_{0}^{z}\left(\widetilde{C^{r}} K^{r}+\widetilde{E}^{r} L^{r}\right) \mathrm{e}^{-\lambda^{r}\left(z-z^{\prime}\right)} \frac{\mathrm{d} \varepsilon_{x}}{\mathrm{~d} z^{\prime}} \mathrm{d} z^{\prime}\right]+\sum_{r}\left[\int_{0}^{z}\left(\widetilde{D^{r}} K^{r}+\widetilde{F}^{r} L^{r}\right) \mathrm{e}^{-\lambda^{r}\left(z-z^{\prime}\right)} \frac{\mathrm{d} \varepsilon_{y}}{\mathrm{~d} z^{\prime}} \mathrm{d} z^{\prime}\right]
\end{aligned}
$$

On the other hand, (16) and (17) are combined to yield

$$
\begin{aligned}
& \sigma_{x}=K^{-3 / 2} \int_{0}^{z} \sum_{r} G^{r} \mathrm{e}^{-\lambda^{r}\left(z-z^{\prime}\right)}\left[(H+F) \frac{\mathrm{d} \varepsilon_{x}}{\mathrm{~d} z^{\prime}}+H \frac{\mathrm{d} \varepsilon_{y}}{\mathrm{~d} z^{\prime}}\right] \mathrm{d} z^{\prime} \\
& \sigma_{y}=K^{-3 / 2} \int_{0}^{z} \sum_{r} G^{r} \mathrm{e}^{-\lambda^{r}\left(z-z^{\prime}\right)}\left[(G+H) \frac{\mathrm{d} \varepsilon_{y}}{\mathrm{~d} z^{\prime}}+H \frac{\mathrm{d} \varepsilon_{x}}{\mathrm{~d} z^{\prime}}\right] \mathrm{d} z^{\prime}
\end{aligned}
$$

Conditions will no be established which will reduce (A1) and (A2) to (A3) and (A4). By considering special cases and equating the two sets of equations, the following relations are obtained

$$
\begin{aligned}
& \tilde{C}^{r} M^{r}+\widetilde{E}^{r} N^{r}=K^{-3 / 2} G^{r}(H+F) \\
& \tilde{D}^{r} M^{r}+\widetilde{F}^{r} N^{r}=K^{-3 / 2} G^{r} H \\
& \widetilde{C}^{r} K^{r}+\widetilde{E}^{r} L^{r}=K^{-3 / 2} G^{r} H
\end{aligned}
$$




$$
\tilde{D}^{r} K^{r}+\widetilde{F}^{r} L^{r}=K^{-3 / 2} G^{r}(G+H)
$$

A condition that makes (A6) equal to (A7) is

$$
\tilde{D}^{r} M^{r}+\widetilde{F}^{r} N^{r}=\tilde{C}^{r} K^{r}+\tilde{E}^{r} L^{r}
$$

The coefficient $G^{r}$ of the kernel function $G(z)$ are then defined from (A6) by

$$
G^{r}=\frac{\widetilde{D^{r}} M^{r}+\widetilde{F}^{r} N^{r}}{\sum_{r}\left(\widetilde{D^{r}} M^{r}+\widetilde{F}^{r} N^{r}\right)} \quad \text { with } \sum_{r} G^{r}=1
$$

Then, from (A5)-(A7),

$$
\begin{aligned}
F & =K^{3 / 2} \sum_{r}\left[\left(\widetilde{C^{r}}-\widetilde{D}^{r}\right) M^{r}+\left(\widetilde{E^{r}}-\widetilde{F}^{r}\right) N^{r}\right] \\
G & =K^{3 / 2} \sum_{r}\left[\left(\widetilde{D^{r}}-\widetilde{C^{r}}\right) K^{r}+\left(\widetilde{F^{r}}-\widetilde{E}^{r}\right) L^{r}\right] \\
H & =K^{3 / 2} \sum_{r}\left[\widetilde{D^{r}} M^{r}+\widetilde{F}^{r} N^{r}\right]
\end{aligned}
$$

Similarly, by comparing (14c) with (16c), it may be found that

$$
G_{x y}(z)=\frac{N D}{K^{3 / 2}} \frac{\sum_{r} D^{r} \mathrm{e}^{-R^{r} z}}{D}=\frac{1}{D} \sum_{r} D^{r} \mathrm{e}^{-R^{r} z}
$$

where

$$
N=\frac{K^{3 / 2}}{D} \quad \text { with } D=\sum_{r} D^{r}
$$

\section{Appendix B}

In the classical theory of plasticity, the yield function is from (2)

$$
2 \phi=(G+H) \sigma_{x}^{2}-2 H \sigma_{x} \sigma_{y}+(F+H) \sigma_{y}^{2}-2 N \sigma_{x y}^{2}
$$

Using the normality condition, the flow rule is

$$
\begin{aligned}
& \mathrm{d} \varepsilon_{x}^{p}=\mathrm{d} \lambda \frac{\partial \phi}{\partial \sigma_{x}}=\mathrm{d} \lambda\left[(G+H) \sigma_{x}-H \sigma_{y}\right] \\
& d \varepsilon_{y}^{p}=\mathrm{d} \lambda \frac{\partial \phi}{\partial \sigma_{y}}=\mathrm{d} \lambda\left[-H \sigma_{x}+(H+F) \sigma_{y}\right]
\end{aligned}
$$




$$
d \varepsilon_{x y}^{p}=\mathrm{d} \lambda \frac{\partial \phi}{\partial \sigma_{x y}}=\mathrm{d} \lambda N \sigma_{x y}
$$

where $\mathrm{d} \lambda$ is a parameter. Equations (B2) may be solved for stress components to yield

$$
\begin{aligned}
\sigma_{x} & =\frac{(H+F) \mathrm{d} \varepsilon_{x}^{p}+H \mathrm{~d} \varepsilon_{y}^{p}}{\mathrm{~d} \lambda M} \\
\sigma_{y} & =\frac{H \varepsilon_{x}^{p}+(G+H) \mathrm{d} \varepsilon_{y}^{p}}{\mathrm{~d} \lambda M} \\
\sigma_{x y} & =\frac{1}{\mathrm{~d} \lambda N} \mathrm{~d} \varepsilon_{x y}^{p}
\end{aligned}
$$

By the substitution of (B3) into (B1), one obtains

$$
\begin{aligned}
\frac{(G+H)}{(\mathrm{d} \lambda M)^{2}}\left[(H+F) \mathrm{d} \varepsilon_{x}^{p}+H \mathrm{~d} \varepsilon_{y}^{p}\right]^{2}- & \frac{2 H}{(\mathrm{~d} \lambda M)^{2}}\left[(H+F) \mathrm{d} \varepsilon_{x}^{p}+H \mathrm{~d} \varepsilon_{y}^{p}\right]\left[(G+H) \mathrm{d} \varepsilon_{y}^{p}+H \mathrm{~d} \varepsilon_{x}^{p}\right] \\
+ & \frac{(H+F)}{(\mathrm{d} \lambda M)^{2}}\left[(G+H) \mathrm{d} \varepsilon_{y}^{p}+H \mathrm{~d} \varepsilon_{x}^{p}\right]^{2}+\frac{2 N}{(\mathrm{~d} \lambda M)^{2}}\left(\frac{M}{N} \mathrm{~d} \varepsilon_{x y}^{p}\right)^{2}=2 \phi
\end{aligned}
$$

It appears natural then to define an equivalent plastic strain increment by

$$
\begin{aligned}
\mathrm{d} \bar{\varepsilon}=\left\{\frac{(G+H)}{K^{3}}\left[(H+F) \mathrm{d} \varepsilon_{x}^{p}+H \mathrm{~d} \varepsilon_{y}^{p}\right]^{2}-\right. & \frac{2 H}{K^{3}}\left[(H+F) \mathrm{d} \varepsilon_{x}^{p}+H \mathrm{~d} \varepsilon_{y}^{p}\right]\left[(G+H) \mathrm{d} \varepsilon_{y}^{p}+H \mathrm{~d} \varepsilon_{x}^{p}\right] \\
& \left.+\frac{(H+F)}{K^{3}}\left[(G+H) \mathrm{d} \varepsilon_{y}^{p}+H \mathrm{~d} \varepsilon_{x}^{p}\right]^{2}+\frac{2 N}{K^{3}}\left(\frac{M}{N} \mathrm{~d} \varepsilon_{x y}^{p}\right)^{2}\right\}^{1 / 2}
\end{aligned}
$$

and to define the equivalent stress $\sigma_{e}$ by

$$
\sigma_{e}=\left(\frac{2 \phi}{K}\right)^{1 / 2}=\left(\frac{3}{2}\right)^{1 / 2}\left\{\frac{(G+H) \sigma_{x}^{2}-2 H \sigma_{x} \sigma_{y}+(F+H) \sigma_{y}^{2}+2 N \sigma_{x y}^{2}}{F+G+H}\right\}^{1 / 2}
$$

so that

$$
\mathrm{d} \bar{\varepsilon}=\mathrm{d} \lambda\left(\frac{M}{K}\right) \sigma_{e}
$$

This definition of equivalent stress is the same as that of Hill (1949). For isotropic materials, (B6) and (B5) reduce, respectively, upon setting $N=3 F=3 G=3 H$, to

$$
\sigma_{e}=\sigma_{x}^{2}-\sigma_{x} \sigma_{y}+\sigma_{y}^{2}+3 \sigma_{x y}^{2}
$$

and

$$
\mathrm{d} \bar{\varepsilon}=\frac{1}{2}\left\{\left[2 \mathrm{~d} \varepsilon_{x}^{p}+\mathrm{d} \varepsilon_{y}^{p}\right]^{2}-\left[2 \mathrm{~d} \varepsilon_{x}^{p}+\mathrm{d} \varepsilon_{y}^{p}\right]\left[2 \mathrm{~d} \varepsilon_{y}^{p}+\mathrm{d} \varepsilon_{x}^{p}\right]+\left[2 \mathrm{~d} \varepsilon_{y}^{p}+\mathrm{d} \varepsilon_{x}^{p}\right]^{2}+3\left(\mathrm{~d} \varepsilon_{x y}^{p}\right)^{2}\right\}^{1 / 2}
$$




$$
=\frac{\sqrt{3}}{2}\left\{\left(\mathrm{~d} \varepsilon_{x}^{p}\right)^{2}+\left(\mathrm{d} \varepsilon_{y}^{p}\right)^{2}+\mathrm{d} \varepsilon_{x}^{p} \mathrm{~d} \varepsilon_{y}^{p}+\left(\mathrm{d} \varepsilon_{x y}^{p}\right)^{2}\right\}^{1 / 2}
$$

\section{Acknowledgement}

The research is supported by the U.S. National Science Foundation (DMI 97-00103) and R.O.C. National Science Council (NSC 85-2811-E002-022).

\section{References}

Barlat, F., Lian, J., 1989. Plastic behavior and stretchability of sheet metals. Part I, a yield function for orthotropic sheet under plane stress conditions. Int. J. Plasticity 5, 51-66.

Barlat, F., Richmond, O., 1987. Prediction of tricomponent plane stress yield surfaces, associated flow and failure behavior of strongly textured f.c.c. polycrystalline sheets. Sci. Engng 95, 15-29.

Gotoh, M., 1977. A theory of plastic anisotropy based on a yield function of fourth order. Int. J. Mech. Sci. 19, 505.

Hill, R., 1948. A theory of yielding and plastic flow of anisotropic metals. Proc. Roy. Soc., London A193, 281-297.

Hill, R., 1979. Theoretical plasticity of textured aggregates. Proc. Camb. Phil. Soc. 85, 179-191.

Hill, R., 1990. Constitutive modelling of orthotropic plasticity in sheet metal. J. Mech. Phys. Solids 38, 405-417.

Im, S., Atluri, S.N., 1987. A study of two finite strain plasticity models: an internal time theory using Mandell's director concept and a general isotropic/kinematic-hardening theory. Int. J. Plasticity 3, 163-191.

Jao, S.Y., Arora, J.S., Wu, H.C., 1991. An optimization approach for material-constant determination for the endochronic constitutive model. Computation Mechanics 8, 25-41.

Lin, S.B., Ding, J.L., 1995. Experimental study of the plastic yielding of rolled sheet metals with the cruciform plate specimen. Int. J. Plasticity 11, 583-603.

Makinde, A., Thibodeau, L., Neale, K.W., 1992. Development of an apparatus for biaxial testing using cruciform specimens. Experimental Mechanics 32, 138.

Mellor, P.B., 1982. Experimental studies of plastic anisotropy in sheet metal. In: Hopkins, Sewel (Eds.), Mechanics of Solids (The Rodney Hill 60th Anniversary Volume), p. 383.

Naruse, K., Dodd, B., Motoki, Y., 1992. Evaluation of yield criteria for planar anisotropy in sheet metal using experimental results. In: Lee, W.B. (Ed.), Advanced Engineering Plasticity Applications. Proceedings of the AsiaPacific Symposium of Advances in Engineering Plasticity and its Applications-AEPA '92, Hong Kong Polytechnical University, Hong Kong, pp. 235-242.

Pearce, R., 1968. Some aspects of anisotropic plasticity in sheet metals. Int. J. Mech. Sci. 10, 995-1005.

Taha, F., Graf, A., Hosford, W., 1995. Plane-strain tension tests on aluminum alloy sheet. J. of Eng. Materials and Technology 117, 168-171.

Valanis, K.C., 1971. A theory of viscoplasticity without a yield surface, Part I: general theory. Archives of Mechanics 23, 517-551.

Valanis, K.C., 1975. On the foundations of endochronic theory of viscoplasticity. Archives of Mechanics 27, 857-868.

Valanis, K.C., 1980. Fundamental consequences of a new intrinsic time measure. Plasticity as a limit of the endochronic theory. Archives of Mechanics 32, 171-191.

Valanis, K.C., Lee, C.F., 1984. Endochronic theory of cyclic plasticity with applications. J. Appl. Mech. 51, 367.

Wagoner, R.H., 1980. Measurement and analysis of plane-strain work hardening. Metallurgical Transactions 11A, 165175.

Wagoner, R.H., Wang, N.-M., 1979. An experimental and analytical investigation of in-plane deformation of 2036-T4 aluminum sheet. Int. J. Mech. Sci. 21, 255-264.

Woodthorpe, J., Pearce, R., 1970. The anomalous behavior of aluminum sheet under balanced biaxial tension. Int. J. Mech. Sci. 12, 341-347. 
Wu, H.C., Lu, J.K., 1995. Further development and application of an endochronic theory accounted for deformation induced anisotropy. Acta Mechanica 109, 11-26.

Wu, H.C., Yang, R.J. 1983. Application of the improved endochronic theory of plasticity to loading with multi-axial strain-path. International Journal of Non-Linear Mechanics 18, 395-408.

Wu, H.C., Yeh, W.C., 1987. Some considerations in the endochronic description of anisotropic hardening. Acta Mechanica 69, 59-76.

Wu, H.C., Yip, M.C., 1980. Strain rate and strain rate history effects on the dynamic behavior of metallic materials. International Journal of Solids and Structures 16, 515-536.

Wu, H.C., Yip, M.C., 1981. Endochronic description of cyclic hardening behavior for metallic materials. ASME Journal of Engineering Materials and Technology 103, 212-217.

Wu, H.C., Hong, H.K., Lu, J.K., 1995a. An endochronic theory accounted for deformation induced anisotropy. Int. J. Plasticity $11,145-162$.

Wu, H.C., Hong, H.K., Shiao, Y.P., 1998. Anisotropic plasticity with application to sheet metals. International Journal of Mechanical Sciences, in press.

Wu, H.C., Lu, J.K., Pan, W.F., 1995b. Endochronic equations for finite plastic deformation and application to metal tube under torsion. International Journal of Solids and Structures 32, 1079-1097. 